\title{
IMPORTANCIA DEL MANEJO DE LA GLUCOSA POSTPRANDIAL EN LA DIABETES TIPO 2. TRATAMIENTO Y NUEVAS TERAPÉUTICAS
}

\author{
SIGNIFICANCE OF POST-PRANDIAL GLUCOSE MANAGEMENT \\ IN TYPE 2 DIABETES. TREATMENT AND NEW THERAPIES
}

Carla Musso, León Litwak, Isaac Sinay

\section{RESUMEN}

El óptimo control glucémico es importante para disminuir el riesgo de complicaciones crónicas en el paciente diabético. Sin embargo hay un alto porcentaje de pacientes tratados con insulina y/o agentes orales que no alcanza los objetivos, particularmente por elevación de la glucemia postprandial.

Tradicionalmente el manejo del paciente con diabetes tipo 2 ha focalizado la terapéutica en el control de la glucemia en ayuno, dado la alta correlación de la misma con la hemoglobina glicosilada. Actualmente hay extensa evidencia para afirmar que la glucemia postprandial cumpliría un rol fundamental en el sub-óptimo control glucémico, con las complicaciones que esto conlleva.

La homeostasis glucémica es compleja de lograr en pacientes con diabetes y la terapéutica debe estar orientada a restablecerla. El objetivo de esta revisión es destacar la importancia del manejo de la glucemia postprandial en diabetes tipo 2, establecer el tratamiento actual de la misma y evaluar cuáles serían las nuevas terapéuticas disponibles.

Palabras clave: diabetes tipo 2, glucemia postprandial y GLP-1.

Revista de la Sociedad Argentina de Diabetes 2014; Vol. 48 (11-21)

Comité de Obesidad y Diabetes. Sociedad Argentina de Diabetes Contacto del autor: Carla Musso

E-mail: mussocar@hotmail.com

Correspondencia: Paraguay 1307, piso $8^{\circ}$ ofic. 74 (C1057AAU), CABA, Argentina Tel./Fax: 4816-2838/4815-1379

Este trabajo fue realizado con el soporte del Laboratorio Sanofi. Los autores declaran no tener conflicto de intereses

\begin{abstract}
Optimal glycemic control is important for reducing the risks of chronic complications in type 2 diabetic patients. However, there are a high percentage of patients treated with insulin and /or oral diabetic drugs that do not achieve their glycated haemoglobin goals, particularly due to elevated post-prandial glucose.

Traditionally, the management of patients with type 2 diabetes has focused on the therapeutic control of fasting plasma glucose, given its high correlation with glycosylated hemoglobin. There is currently extensive evidence to support that the postprandial glucose has a fundamental role in the sub-optimal glycemic control, with the complications that entails.

The glycemic homeostasis is complex to achieve in patients with diabetes, and the therapy should be aimed at reseting it. The principal objective of this review is to emphasize the importance of managing post-prandial glucose in Type 2 diabetic patients, establish the current treatment and evaluate what would be the new therapeutics available.
\end{abstract}

Key words: Type 2 diabetes, post-prandial glucose and GLP-1.

Revista de la Sociedad Argentina de Diabetes 2014; Vol. 48 (11-21)

\section{INTRODUCCIÓN}

Se ha demostrado que pacientes con diabetes tipo 2 (DM2) con mal control de la enfermedad tienen riesgo incrementado para desarrollar complicaciones microvasculares (retinopatía, neuropatía y nefropatía) así como macrovasculares (enfermedad coronaria y arteriopatía de miembros inferiores). Los estudios Diabetes Control and Complications Trial (DCCT) ${ }^{1}$ y UK Prospective Diabetes Study (UKPDS) ${ }^{2}$ evidenciaron que la mejoría del control glucémico, determinado por la hemoglobina glicosilada ( $\mathrm{HbA} 1 \mathrm{c})$, reduce los eventos microvasculares. Sin embargo el efecto de este control sobre los eventos macrovasculares aún no está claro.

Recientes hallazgos sugieren que entre un 40 y un $50 \%$ de los pacientes tratados con insulina y/o agentes orales no alcanza el objetivo de $\mathrm{HbA}_{1} \mathrm{c}^{3}$, 
relacionando este hecho a un mal control de la glucemia postprandial (GPP). La hiperglucemia postprandial es una de las anormalidades iniciales de la homeostasis de la glucosa en la diabetes tipo 2.

En pacientes con DM2 se encontró que la importancia de la glucosa plasmática en ayunas (GPA) y la GPP es relativa según el control metabólico. Con $\mathrm{HbA} 1 \mathrm{c}<7,3 \%$, la GPP contribuye en un $70 \%$ y la GPA un $30 \%$. Sin embargo cuando la $\mathrm{HbA} 1 \mathrm{c}$ $<10 \%$, la GPA tiene un alto impacto en un $70 \%$ y la GPP en un $30 \%{ }^{4}$. Establecer la terapéutica de acuerdo al mecanismo afectado permitirá lograr el objetivo glucémico ideal para cada paciente.

En los últimos años las recomendaciones para el tratamiento de la DM2 han destacado cada vez más la importancia del control de GPP: la Federación Internacional de Diabetes (IDF) recomienda un objetivo de GPP de $\leq 140 \mathrm{mg} / \mathrm{dl}^{5}$. Si bien hay un debate instalado sobre la importancia del control de la GPP6, ya que los objetivos resultan poco realistas e incluso se podrían asociar al incremento de hipoglucemia7, las recomendaciones científicas sobre el control de la GPP son claras. La mayoría de los pacientes con diabetes tipo 2 tiene GPP elevada, incluso cuando la $\mathrm{HbA} 1 \mathrm{c}$ es satisfactoria $(<7 \%)$. Datos recientemente publicados evidenciaron que niveles elevados de GPP se han encontrado en el $74 \%$ de los individuos con $\mathrm{DM}^{8}$. Por otro lado, la GPP es un factor independiente de enfermedad cardiovascular en pacientes con DM2 ${ }^{9}$.

\section{Homeostasis glucémica de ayuno en pacientes con DM2}

La DM2 presenta un estado de insulinorresistencia (IR) tanto periférica como central (hepática), con un déficit progresivo en la secreción de insulina por parte de las células beta. Esto se acompaña, además, por una secreción aumentada de glucagón. Este conjunto de alteraciones determina un incremento de la producción hepática de glucosa, tanto nocturna como postprandial ${ }^{10}$.

\section{Homeostasis glucémica prandial normal}

Cuando la glucosa, contenida en los alimentos mixtos, es absorbida rápidamente a nivel intestinal se produce un secuestro pequeño de la misma en el área esplácnica y la mayor parte de esta carga pasa a la circulación arterial periférica apareciendo en la misma en relación a la velocidad de vaciamiento gástrico. Se produce un primer pico de secreción de insulina entre los 30 y 40 minu- tos que siguen a una ingestión y luego un segundo pico más tardío entre los 120 y 150 minutos (Figura 1). Sin embargo, la glucemia no vuelve a los estados de ayuno hasta 5 horas después de la ingesta. A esto se denomina período postabsortivo. Es decir que una vez generada la hiperglucemia post ingesta, el retorno a valores similares al ayuno ocurre en aproximadamente ese tiempo generando una curva muy estable y reproducible de concentración de glucosa en relación a la liberación de insulina ${ }^{10}$.

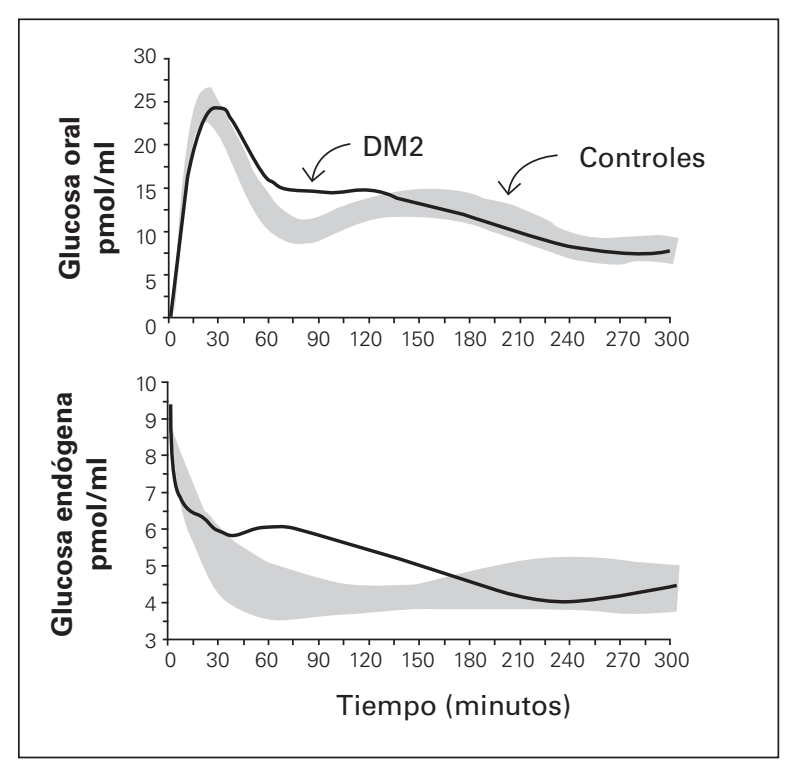

Figura 1: Comportamiento de la glucemia en pacientes DM2 y controles luego de la ingesta de comida. Adaptado de Ferrarini E. Physiology of glucose homeostasis and insulin therapy in type 1 and type 2 diabetes. Endocrinol. Metab. Clin. N. Am. 2012; 41:25-39.

Al ingreso del bolo alimenticio, la glucosa principalmente y los aminoácidos en menor medida estimulan a las células beta para liberar insulina (INS). La liberación se produce rápidamente. La relación portosistémica de la concentración de INS es proporcionalmente similar a la que se observa en estados de ayuno (dos a cuatro veces) pero la concentración pre-hepática absoluta de la misma es elevada (130-170 u U/ml). En pocos minutos este gradiente cae a menos de 1,5 veces indicando que hay un pasaje de INS a la periferia. Por lo tanto, en cuanto a INS, se observa un primer pico, luego un segundo pico y finalmente un descenso paulatino hasta llegar al estado de ayuno. Se distingue claramente el estado de ayuno de los períodos postprandiales y postabsortivos ${ }^{10}$. 
El área bajo la curva de INS dependerá del contenido calórico de la ingesta. A mayor cantidad de calorías mayor es el estímulo para la liberación de INS y durante más tiempo (Figura 2).

Por el contrario, la concentración de glucosa (GLC) desciende significativamente luego de la ingesta alimentaria. La llegada del bolo alimenticio al tracto gastrointestinal gatilla un complejo sistema de gastro-entero-hormonas o incretinas cuyo representante más conocido es el glucagón like peptide 1 (GLP-1) que genera la liberación de INS, disminuye la secreción de GLC suprimiendo en más de un $50 \%$ la producción hepática de glucosa coincidiendo con un incremento del índice INS/ GLC. Esta situación se mantiene durante aproximadamente las 5 horas posteriores a la ingesta. El incremento de INS estimula, por otra parte, la captación de glucosa por los tejidos periféricos insulinodependientes. Es importante destacar que GLP-1, además de las funciones descriptas, enlentece el vaciamiento gástrico y produce disminución central del apetito ${ }^{10}$.

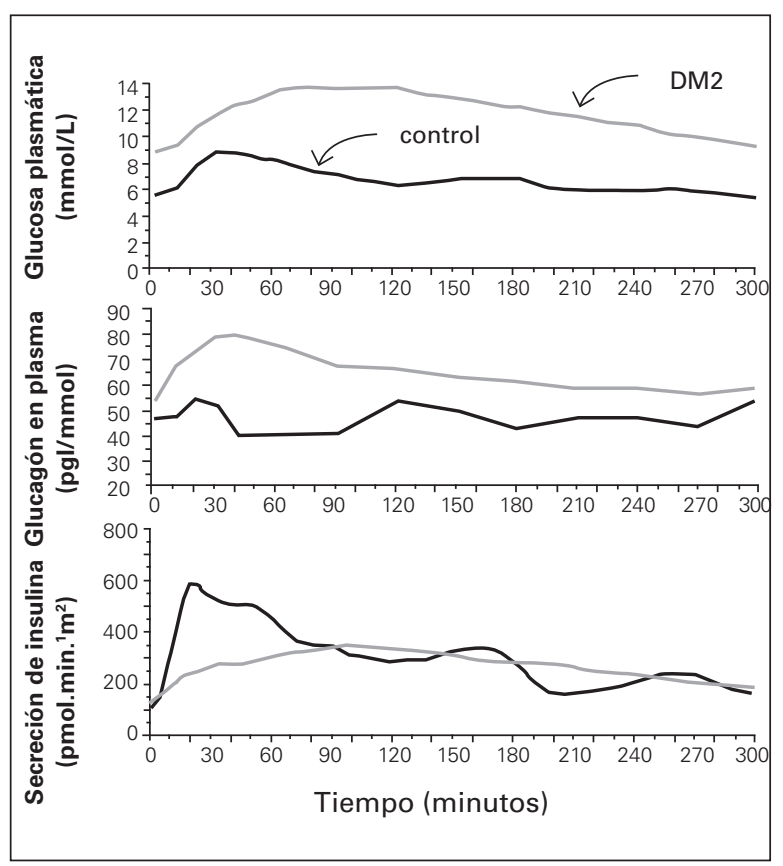

Figura 2: Concentración de glucosa, glucagón y secreción de insulina en pacientes DM2. Adaptado de Ferrarini E. Physiology of glucose homeostasis and insulin therapy in type 1 and type 2 diabetes. Endocrinol. Metab. Clin. N. Am. 2012; 41: 25-3910.

\section{Homeostasis glucémica prandial en pacientes con DM2}

En estos pacientes se observa una disfunción de la respuesta incretínica (GLP-1) evidenciada por la disminución en la secreción de INS y la falta de inhibición en la secreción de GLC. Esto provoca una falta de inhibición en la producción de glucosa endógena hepática generando hiperglucemia postprandial y postabsortiva significativamente incrementada en relación a individuos no diabéticos. El área bajo la curva de glucosa está significativamente aumentada y extendida (Figura 2). En situación de descompensación glucémica, se observa tanto en DM1 (por déficit de INS) como en DM2 (por déficit de acción o por secreción insuficiente de INS) un estado de hiperglucemia importante y duradero.

En base a lo descripto surge claramente que tanto IR como insulinopenia relativa en el paciente con DM2 como la falta total de INS en el DM1, determinan un estado de descontrol glucémico caracterizado por hiperglucemia de ayuno (basal) e hiperglucemia postprandial y postabsortiva. Un mejor conocimiento de estas alteraciones generará en el futuro un tratamiento más racional y eficiente de estas alteraciones ${ }^{10}$.

\section{Hiperglucemia postprandial: estrés oxidativo, inflamación y disfunción endotelial}

Se demostró que las fluctuaciones de la glucosa durante períodos postprandiales mostraban un efecto detonante más concreto sobre el estrés oxidativo que la hiperglucemia crónica permanente en personas con DM2, en comparación con personas sin diabetes $^{11}$. También se ha observado ${ }^{12}$ que las personas con DM2 e hiperglucemia postprandial estaban expuestas a períodos de estrés oxidativo inducidos por las comidas a lo largo del día'2 (Figura 3 ).

Se han registrado elevados niveles de moléculas de adhesión que juegan un importante papel en la iniciación de la aterosclerosis en personas con diabetes ${ }^{13}$. Ceriello y col $^{14,15}$ estudiaron los efectos de tres comidas distintas (comida con un alto contenido de grasas, $75 \mathrm{~g}$ de glucosa aislados, comida con un alto contenido en grasas sumada a $75 \mathrm{~g}$ de glucosa) en pacientes con DM2 y 20 personas sin diabetes. Los resultados demostraron un efecto independiente y acumulativo de la hipertrigliceridemia e hiperglucemia postprandial sobre los niveles en plasma de MAIC-1, MACV-1 y E-selectina.

La hiperglucemia aguda en respuesta a una 
carga glucémica por vía oral en personas con una tolerancia normal a la glucosa, ATG o diabetes tipo 2 suprimió rápidamente la vasodilatación dependiente del endotelio y la alteración de la liberación de óxido nítrico endotelial ${ }^{16}$. Otros estudios demostraron que la hiperglucemia aguda en personas normales altera la vasodilatación dependiente del endotelio ${ }^{17}$ y podría activar la trombosis, aumentar los niveles circulantes de moléculas de adhesión solubles y prolongar el intervalo $Q T^{18}$.

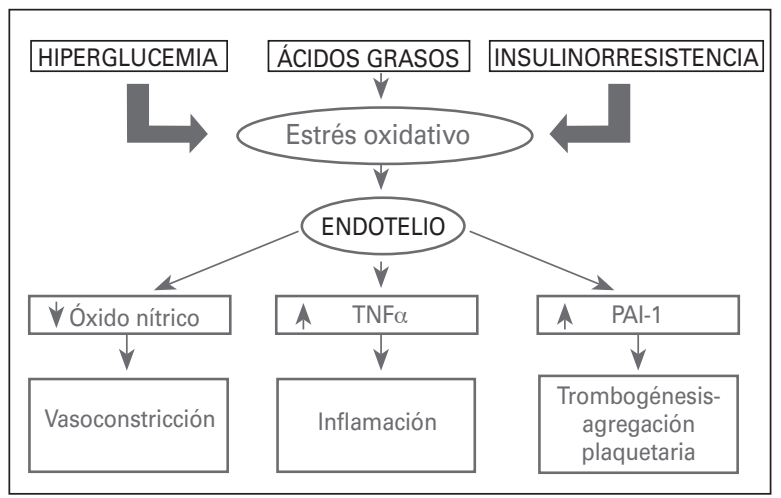

Figura 3: Estrés oxidativo en pacientes con DM2.

\section{Hiperglucemia postprandial y riesgo cardiovascular}

Como se mencionó anteriormente, la GPP es un predictor independiente de enfermedad cardiovascular. Varios mecanismos están relacionados con las lesiones vasculares; numerosos estudios respaldan la hipótesis de que exista una relación causal entre la hiperglucemia postprandial y el estrés oxidativo. La hiperglucemia, los ácidos grasos libres y la resistencia a la insulina potencian el estrés oxidativo, la activación de la proteína quinasa-C (PKC) y la activación de los receptores de los productos finales avanzados de glucosilación (RAGE), lo cual genera vasoconstricción, inflamación y trombosis (Figura 3).

La hiperglucemia aguda y la variabilidad glucémica parecen jugar un papel importante en este mecanismo. Un estudio examinó la apoptosis en células endoteliales de las venas umbilicales humanas mediante cultivos celulares que se sometieron a un estado estable y alternando las concentraciones de glucosa. También se demostró que la variabilidad de los niveles de glucosa podría ser más dañina que la concentración elevada de glucosa en forma constante.

Los estudios Análisis Epidemiológico Colaborativo de los Criterios de Diagnóstico de Diabetes en
Europa (Diabetes Epidemiology Collaborative Analysis of Diagnostic Criteria in Europe, DECODE) y el Análisis Epidemiológico Colaborativo de los Criterios de Diagnóstico de Diabetes en Asia (Diabetes Epidemiology Collaborative Analysis of Diagnostic Criteria in Asia, DECODA) ${ }^{19}$ analizaron datos de valores base y -a las 2 horas de realizar una prueba de sobrecarga de glucosa procedente de estudios de cohorte en los cuales participó un gran número de varones y mujeres de origen europeo y asiático- revelaron que la glucemia a las 2 horas predecía mejor la enfermedad cardiovascular y la mortalidad por cualquier causa que la glucemia en ayunas.

Levitan y sus colegas ${ }^{20}$ realizaron un metaanálisis de 38 estudios prospectivos y confirmaron que la hiperglucemia, en personas no diabéticas, estaba asociada a un aumento del riesgo de enfermedad cardiovascular fatal y no fatal, con una relación similar entre eventos y glucemia en ayunas y 2 horas postprandial. En el análisis, 12 estudios que registraron valores de glucosa en plasma en ayunas y seis estudios que registraron la glucemia postprandial, permitieron el cálculo de las curvas de respuesta según la dosis. Los accidentes cardiovasculares aumentaron de modo lineal sin que hubiese un umbral para la glucosa en plasma postprandial a las 2 horas, mientras que la glucosa en plasma en ayunas mostró un posible efecto umbral a los $5,5 \mathrm{mmol} / \mathrm{l}(99 \mathrm{mg} / \mathrm{dl})$. De modo similar, en el estudio longitudinal entre los mayores de Baltimore (Baltimore Longitudinal Study of Aging $)^{21}$, que realizó un seguimiento a 1.236 varones y mujeres durante un período medio de 13,4 años a fin de determinar la relación entre la glucosa en plasma en ayunas y la glucosa en plasma postprandial a las 2 horas y la mortalidad por cualquier causa, lo que determinó que esta última aumentaba de manera importante cuando el nivel de glucosa en plasma en ayunas superaba los 6,1 $\mathrm{mmol} / \mathrm{l}(110 \mathrm{mg} / \mathrm{dl})$, pero no con niveles de glucosa en plasma en ayunas más bajos. Sin embargo, el riesgo aumentaba notablemente cuando los niveles de glucemia postprandial a las 2 horas superaban los $7,8 \mathrm{mmol} / \mathrm{l}(140 \mathrm{mg} / \mathrm{dl})$.

Las observaciones también se amplían a personas con diabetes, siendo la glucemia postprandial un predictor más sólido de accidentes cardiovasculares que la glucosa en plasma en ayunas en la DM2, especialmente en mujeres.

Para analizar esta eventual asociación revisaremos la evidencia sobre los valores glucémicos 2 
horas después de una prueba de tolerancia oral a la glucosa (PTOG) como predictores de enfermedad cardiovascular (ECV).

Con esa finalidad haremos una revisión sucinta de los estudios FUNAGATA22, DECODE ${ }^{19}$, Framingham Offspring Study ${ }^{23}$ y de un metaánalisis publicado en $2004^{20}$ con numerosos estudios prospectivos realizados hasta ese momento.

En el estudio FUNAGATA ${ }^{22}$ el objetivo era determinar si la categoría de glucemia alterada en ayunas (GAA) definida por el Comité de Expertos de la American Diabetes Association (ADA) poco antes de dicha investigación era un factor de riesgo para ECV. La población estudiada fue de 2.016 personas con tolerancia normal a la glucosa (TNG), 382 con tolerancia alterada a la glucosa oral (TAG) y 283 con DM2, que fueron luego reclasificadas de acuerdo a la propuesta de la ADA en 2.307 con TNG, 155 con GAA y 189 con DM2.

La cohorte poblacional fue seguida durante siete años y evidenció que la TAG era un factor de riesgo para ECV pero que la GAA no lo era (Tabla 1). Es de destacar que el Hazard Ratio (HR) era sensiblemente parecido para TAG y DM2.

\begin{tabular}{|l|r|r|r|c|}
\hline $\begin{array}{c}\text { Muerte } \\
\text { CV según } \\
\text { criterio }\end{array}$ & $\begin{array}{c}\text { Número de } \\
\text { muertes }\end{array}$ & HR & $\mathbf{( 9 5 \% ~ C l )}$ & $\mathbf{p}$ \\
\hline OMS 1985 & & & & \\
\hline TAG & 11 & 2,219 & $(1,079-1,050)$ & 0,0001 \\
\hline DM2 & 11 & 2,274 & $(1,069-4,838)$ & 0,0309 \\
\hline ADA 1997 & & & & \\
\hline GAA & 3 & 1,136 & $(0,345-3,734)$ & 0,8342 \\
\hline DM2 & 3 & 1,136 & $(0,345-3,734)$ & 0,8342 \\
\hline
\end{tabular}

Tabla 1: Análisis de Cox para las categorías comparadas con tolerancia normal a la glucosa (TNG).

El Estudio DECODE fue un seguimiento prospectivo de 8,8 años de 11 cohortes europeas que realizaron PTOG (en la base 20.707 eran no diabéticos, 796 tenían diabetes conocida, y 1.011 fueron diagnosticados como diabéticos: 355 por GPA, 355 post PTOG y 301 por ambos criterios); 2.557 presentaban TAG.

La conclusión fue que la glucemia post ingesta oral de glucosa era mejor predictor de muerte por todas las causas y de enfermedad coronaria que la glucemia en ayunas. También se observó un riesgo aumentado (ajustado por la GPA en el análisis multivariado) para eventos CV y muerte CV en TAG (Tabla 2).

\begin{tabular}{|l|c|c|c|}
\hline & $\begin{array}{c}\text { Diabetes por } \\
\text { PTOG } \\
\text { HR } \mathbf{( 9 5} \% \mathbf{C l})\end{array}$ & $\begin{array}{c}\text { Diabetes por GA } \\
\text { HR } \mathbf{( 9 5} \% \mathbf{C I})\end{array}$ & $\begin{array}{c}\text { TAG } \\
\text { RR* ajuste } \\
\text { multivariado }\end{array}$ \\
\hline $\begin{array}{l}\text { Muerte por } \\
\text { todas las } \\
\text { causas }\end{array}$ & $1,73(1,45-2,06)$ & $1,21(1,01-1,44)$ & $1,37(1,25-1,51)$ \\
\hline $\begin{array}{l}\text { Muerte car- } \\
\text { diovascular }\end{array}$ & $1,40(1,02-1,92)$ & $1,20(0,88-1,64)$ & $1,32(1,12-1,56)$ \\
\hline $\begin{array}{l}\text { Enfermedad } \\
\text { coronaria }\end{array}$ & $1,56(1,03-2,36)$ & $1,09(0,71-1,67)$ & $1,27(1,03-1,58)$ \\
\hline
\end{tabular}

Tabla 2: Incidencia de muertes en TAG y GAA. *Ajuste para edad, centro, sexo, colesterol, IMC, PA, tabaco y glucemia plasmática en ayunas.

En el Framingham Offspring Study, en un seguimiento prospectivo de cuatro años de personas sin diabetes diagnosticada, se demostró que tanto la GPA, la GPP y la HbA1c incrementaban el riesgo de ECV aún teniendo en cuenta los factores de riesgo (FR) tradicionales no glucémicos. El RR aumentaba en los cuartilos crecientes de glucemia 2 horas post carga de glucosa y es de destacar que cuando se tenía en cuenta este valor de la GPP, tanto la GA como la HbA1c dejaban de ser predictores independientes de ECV. Cuando el modelo de análisis tomaba en conjunto la GA y GPP a las 2 horas en la PTOG, el RR para ECV era no significativo para GA y sí lo era para GPP a las 2 horas en la PTOG. Sin embargo el RR era de 1,1 (95\% Cl 1,02-1,30), por lo tanto este estudio plantea que es un valor predictivo pequeño comparado con los FR no glucémicos tradicionales que continúan siendo el mejor criterio de identificación de los sujetos en los que se debe intervenir para reducir el riesgo de ECV.

En el metaanálisis anteriormente mencionado ( 195.000 personas en 29 estudios con una media de 12 años de seguimiento) se analizó exclusivamente si la hiperglucemia no diabética era un FR para ECV. La glucemia más elevada 2 horas PTOG mostró una relación lineal para riesgo de ECV (RR 1,19,95\% Cl 1,07-1,32) comparando los niveles más elevados de glucemia (150-194 mg/dl) vs. los más bajos (69-107 mg/dl) ajustando para los otros FR de ECV y con criterios estrictos para exclusión de personas diabéticas. Los autores destacaron que en esta relación lineal hay un efecto "umbral" con GPA cercano a $100 \mathrm{mg} / \mathrm{dl}$. El riesgo parece ser mayor en mujeres que en hombres. Este metaanálisis concluyó que la glucemia era un FR para ECV en individuos sin diabetes.

Es de interés mencionar un estudio del subgrupo de normoglucémicos de DECODE (GPA $<110$ mg/dl 
y glucemia 2 horas PTOG $<140 \mathrm{mg} / \mathrm{dll}$ ) en el cual se aprecia que la glucemia 2 horas PTOG se asocia con un aumento de muerte CV comparando aquellos que en 2 horas PTOG mantienen valores mayores que la GPA vs. aquellos que a las 2 horas retornan a los valores de ayunas o menores. El exceso de riesgo se apreciaba también para la diferencia en GPA y la de 2 horas PTOG expresada como una variable continua. Este hecho no se percibía para GPA ${ }^{19}$.

Debemos considerar que la respuesta glucémica a una situación experimental y no fisiológica como es la PTOG y su eventual impacto, no es extrapolable a lo que ocurra con la glucemia postprandial (GPP) que es definida por niveles glucémicos luego de la ingesta de alimentos. Analizaremos, entonces, algunas evidencias sobre asociaciones de niveles de GPP y ECV. En otro lugar de esta revisión, se analiza la definición glucémica de hiperglucemia postprandial (HGPP), pero recordemos que no hay acuerdo sobre el nivel que la define ni el momento oportuno post comida con valores tan divergentes como $140 \mathrm{mg} / \mathrm{dl}$ para algunos y $180 \mathrm{mg} / \mathrm{dl}$ tomados en muestras plasmáticas de 1, 2 ó 3 horas post ingesta ${ }^{4,24,25}$.

Ya en 1996 el Diabetes Intervention Study ${ }^{26}$ en un seguimiento de 1.139 diabéticos con diagnóstico reciente se observó que la GPP era un factor de riesgo para infarto de miocardio e independiente para muerte por todas las causas, considerando PA, tabaco, sexo, edad y triglicéridos pero no se incluyó HbA1c en el estudio.

En el estudio de Luigi Gonzaga9 ${ }^{9}$, se analiza si la GPP predice muerte por todas las causas y tiempo al primer evento CV en 505 pacientes con DM2 seguidos durante 14 años. Se tomaron cinco parámetros glucémicos $(\mathrm{HbA} 1 \mathrm{c}, \mathrm{GPA}$ y precena, y GPP 2 horas post desayuno y post almuerzo). Considerándolos en conjunto, los únicos predictores fueron la GPP post almuerzo y la HbA1c (Tabla 3).

\begin{tabular}{|l|c|c|}
\hline & $\begin{array}{c}\text { Glucemia } 2 \text { hs. post } \\
\text { almuerzo HR } \mathbf{( p )}\end{array}$ & $\begin{array}{c}\text { HbA1c } \\
\text { HR (p) }\end{array}$ \\
\hline Tiempo a eventos CV & $1.507(=0,010)$ & $1.792(=0,002)$ \\
\hline $\begin{array}{l}\text { Mortalidad por todas } \\
\text { las causas }\end{array}$ & $1.885(=0,0001)$ & $1.907(=0,0002)$ \\
\hline
\end{tabular}

Tabla 3: Impacto CV y en mortalidad de la glucemia post glucosa oral.

Cuando esos dos parámetros se consideraron en conjunto con los principales FR no glucémicos, su capacidad de predicción se mantenía (Tabla 4*).

\begin{tabular}{|l|c|c|}
\hline & $\begin{array}{c}\text { Glucemia } 2 \text { hs. post } \\
\text { almuerzo HR (p) }\end{array}$ & $\begin{array}{c}\text { HbA1c } \\
\text { HR }(\mathbf{p})\end{array}$ \\
\hline Tiempo a eventos CV & $1.452(=0,021)$ & $1.732(=0,004)$ \\
\hline $\begin{array}{l}\text { Mortalidad por todas } \\
\text { las causas }\end{array}$ & $1.846(=0,001)$ & $1.896(=0,004)$ \\
\hline
\end{tabular}

Tabla 4: Glucemia post ingesta y mortalidad.

* Análisis de Cox introduciendo sexo, edad, duración DT, tabaco, IMC, PAS, PAD, LDLc, HDLc, triglicéridos, creatinina, albuminuria, HbA1c y GPP 2 horas post almuerzo.

Se debe tener en cuenta en el análisis de este estudio que la población tratada con dieta exclusiva era elevada $(45 \%)$ y el empleo de insulina relativamente bajo (12\%) para una población que al finalizar los 14 años de seguimiento tenía 23 años de duración conocida de la DM2 (9,4 años de duración al comienzo de la observación).

La conclusión más importante de este estudio con un seguimiento prolongado era que la $\mathrm{HbA} 1 \mathrm{c}$ y la GPP (y no la GPA) predicen ECV y mortalidad por todas las causas también cuando se consideran en conjunto con los principales FR no glucémicos.

Es interesante recordar el estudio de Esposito en el cual los autores siguieron durante 12 meses a una población diabética en la que, con dos terapéuticas diferentes, mantuvieron un descenso final de $\mathrm{HbA} 1 \mathrm{c}$ similar $(-0,9 \%)^{27}$. Se diferenciaron ambas ramas en la cual una tuvo valores de GPP menores que la otra al terminar el estudio $(148+28 \mathrm{mg} / \mathrm{dl}$ vs. $18+32 \mathrm{mg} / \mathrm{dl}, \mathrm{p}<0,01)$. Se observó que había una regresión del espesor íntima/media carotideo, predefinido como una reducción mayor de 0,020 $\mathrm{mm}$ en un porcentaje mayor de sujetos en la rama con menor GPP $(52 \%$ vs. $18 \%, p<0,01)$. Esto se asociaba con valores significativamente más bajos de interleuquina 6 y de proteína $C$ reactiva.

Como conclusión, la glucemia elevada post ingesta de glucosa oral parece ser un factor de riesgo independientemente para enfermedad cardiovascular, como así también la GPP. En ambos casos debe entenderse que otros parámetros glucémicos alterados como GA y HbA1c también son factores de riesgo para enfermedad cardiovascular.

\section{Enfoque terapéutico tradicional de hiperglucemia postprandial}

Las drogas hipoglucemiantes con mayor impacto postprandial son el acarbose y las meglitinidas.

El acarbose -o inhibidor de las alfa glucosidasas- cuyo mecanismo de acción es la inhibición 
selectiva de las $\alpha$-glucosidasas en el borde en cepillo de intestino delgado, retrasa la absorción de los hidratos de carbono. Éstos se absorben a nivel distal y en forma más tardía disminuyendo la GPP.

El acarbose provoca molestias gastrointestinales como diarreas, meteorismo y dolor abdominal marcado que dificulta su prescripción.

The STOP-NIDDM Trial fue el primer estudio en investigar la relación entre el descenso de GPP con acarbose y el desarrollo de eventos CV. Los resultados del estudio demostraron un descenso significativo del riesgo CV e HTA en el grupo de personas con tolerancia alterada a la glucosa, tratado con acarbose ${ }^{28}$.

La disminución en la GPP con acarbose se asoció a un 49\% de reducción de RR en desarrollo de eventos CV HR 0,51 (IC 95\% 0,01-0,72: P = 0,3) y HTA (HR, 0,62; 95\% IC, 0,45-0,86; $P=0,04$ ).

Otro estudio, The Early Diabetes Intervention Program, recientemente publicado, demostró que la progresión del espesor íntima/media (EIM) marcador subrogante de RCV riesgo CV se redujo en el grupo acarbose $(p=0,047)$ cuando se lo comparó con placebo 29 .

\section{Meglitinidas: repaglinida y nateglinida}

Ambas moléculas son compuestos no sulfonilureicos derivados del ácido benzoico. El mecanismo de acción es similar al de las sulfonilureas (SU), ejerciendo su actividad hipoglucemiante al unirse a los receptores de m SUR1/kir 6,2 en las células $\beta$ pancreáticas.

Tienen un efecto hipoglucemiante más corto y rápido que las SU, razón por la cual permiten controlar de manera más eficiente la glucemia GPP. La nateglinida tiene duración de acción más corta y menor riesgo de hipoglucemia que la repaglinida. Ambas se absorben rápidamente y producen aumento de la secreción de insulina a los 30 minutos $^{30}$.

Son de elección en pacientes con hipoglucemias tardías con SU o con hábitos irregulares de comida.

Reducen la A1c HbA1c 0,5-1\% con menor tasa de hipoglucemias y menor ganancia de peso que las SU. Se recomienda ingerirlas 30 minutos antes de la comidas, siendo ésta una de las causas de la baja adherencia de los pacientes a esta medicación. En caso de no ingerir alimentos, se debe suspender su toma.

El estudio NAVIGATOR (Nateglinide and Valsartan in Impaired Glucosa Tolerance Outcomes Research) que incluye nateglinide, fue diseñado para evaluar si las drogas que bloquean el sistema renina angiotensina reducen el riesgo de DBT y eventos CV en pacientes con ITG. En el seguimiento de los pacientes durante cinco años, nateglinida no tuvo impacto en ninguno de los objetivos estudiados y el valsartán se asoció únicamente a una reducción de nuevos casos de diabetes, aunque esto podría tener más de una interpretación ${ }^{31}$.

\section{Nuevas alternativas terapéuticas}

El defecto incretínico al que se hizo referencia previamente con la insuficiente estimulación por parte del GLP-1 endógeno de la célula beta para la secreción de insulina así como también de la supresión de la secreción de glucagón por las células alfa, promueve la hiperglucemia postprandial ${ }^{32}$. La disfunción del mecanismo incretínico forma parte de la evolución de la enfermedad y se correlaciona con los años de diagnóstico de la diabetes. Estudios realizados con análogos del receptor de GLP-1 (GLP-1 RA) han demostrado tener efecto sobre la saciedad, el vaciamiento gástrico y por consiguiente en la GPP y el descenso de peso. El impacto diferente sobre la glucemia postprandial y sobre el retraso en el vaciamiento gástrico están claramente relacionados con la duración de la vida media, razón por la cual se los divide en:

- GLP-1 RA prandiales de acción corta: exenatide, se administra dos veces por día, y lixisenatide una vez por día. Estos análogos tienen un efecto sobre el descenso de la GPP pronunciado y en el retraso del vaciamiento gástrico.

- Exenatide. Es un análogo de exendina-4, un péptido de 39 aminoácidos, aislado de la saliva del monstruo de Gila, que tiene una secuencia similar al GLP-1 nativo y ejerce un efecto agonista sobre el receptor de GLP-1. Su vida media es de 2 horas y requiere de dos inyecciones subcutáneas diarias. En individuos con DM2 en tratamiento subóptimo con ADOs, el agregado de exenatide redujo la $\mathrm{HbA} 1 \mathrm{c}$ en $0,8-0,9 \%$, con una reducción de la GPP del área bajo la curva entre el 34 y $87 \%$ según la combinación de ADOs. En estudios realizados comparando exenatide más metformina vs. sitagliptina más metformina, la reducción de la GPP fue de 6,2 mmol/l vs. 2,1 $\mathrm{mmol} / \mathrm{l}(\mathrm{p}<0,0001)^{33-34}$. Similares resultados fueron hallados en estudios comparados con TZD (tiazolidinedionas). En cuanto a la combinación de exenatide con insulina glargina o insulina bifásica, el mayor descenso de GPP se logró con insulina 
bifásica, mientras que con glargina disminuyeron los valores de glucemia en ayunas ${ }^{32}$.

- Lixisenatide. Es un análogo de exendina 4, de 44 aa con un agregado en la cadena $\mathrm{c}$ terminal. Los análogos del GLP 1 de acción corta ejercen sus efectos sobre la GPP a través del retraso del vaciamiento gástrico, enlenteciendo el aporte de glucosa a través del duodeno. La corta vida media (3 horas) de lixisenatide, sumada a su alta afinidad por el receptor de GLP1 (cuatro veces más que el GLP1 nativo), podría dar un efecto más sostenido sin producir taquifilaxia ${ }^{35}$.

A partir de los estudios del programa Get-Goal donde participaron más de 4.500 pacientes, se evaluaron la eficacia y seguridad de lixisenatide asociados a hipoglucemiantes y/o insulina en los que se demostró descenso significativo de $\mathrm{HbA} 1 \mathrm{c}$, modestos efectos beneficiosos sobre el peso comparados con placebo y pronunciado efecto en el descenso de glucemia postprandial. Los efectos adversos fueron náuseas $22 \%$ vs. $14 \%$ con placebo; no se registraron hipoglucemias y el descenso de peso fue de $1 \mathrm{~kg}$ en 24 semanas $^{36}$.

Get-goal $X^{37}$ es un estudio randomizado de no inferioridad donde se compararon eficacia (principalmente descenso de HbA1c) y seguridad (hipoglucemias, tolerancia gastrointestinal) de exenatide $10 \mu \mathrm{g}$ administrado dos veces por día vs. lixisenatide $20 \mu$ una vez por día, en pacientes con DM2 con mal control glucémico en tratamiento con metformina. El descenso de $\mathrm{HbA} 1 \mathrm{c}$ fue similar en ambos grupos, demostrándose no inferioridad de lixisenatide en eficacia comparado con exenatide. En el grupo lixisenatide se presentó menor tasa de hipoglucemia sintomática (2,5 vs. $7,9 \%$; $P<0,05)$ y menores episodios de intolerancia gastrointestinal, especialmente náuseas $(24,5$ vs. $35,1 \% ; P<0,05)$ con respecto a exenatide ${ }^{37}$.

Otro estudio de lixisenatide contra placebo de 28 días de duración -en DM2, en tratamiento con al menos 2 ADOs- evaluó la tasa de vaciamiento gástrico y el control de la glucemia. Para la determinación de la tasa de vaciamiento gástrico se utilizó la técnica del ácido C-octanoico, que es la mejor técnica de respiración radiomarcada. Lixisenatide se asoció a reducciones significativas en el área bajo la curva AUC de las GPP. Así también el tiempo de vaciamiento gástrico fue significativamente mayor con lixisenatide comparado con placebo, razón por la cual se concluyó que lixisenatide $20 \mu \mathrm{g}$ una vez al día redujo las exclusiones glucémicas post- prandiales en pacientes con $\mathrm{DM} 2^{35}$. Actualmente se está desarrollando el estudio ELIXA (Evaluation of Cardiovascular Outcomes in Patients with Type 2 Diabetes after Acute Coronary Syndrome During Treatment with Lixisenatide) que evalúa el potencial beneficio cardiovascular de lisixenatide en pacientes diabéticos tipo 2 que han presentado un síndrome coronario agudo (SCA) recientemente.

- Análogos de GLP-1 no prandiales: liraglutide, albiglutide, semaglutide y exenatide Lar (de acción prolongada).

Los análogos no-prandiales lograron por diferentes mecanismos extender su acción retrasando su absorción a nivel subcutáneo. El exenatide Lar tiene un sistema de microesferas que permite colocarlo una vez por semana. El liraglutide tiene una cadena de ácido palmítico en Lys 26 y un reemplazo de lisina por arginina. Albiglutide es un dimero de GLP-1 que se une a albumina, y semaglutide un análogo monoacetilado de GLP-1, ambas modificaciones confieren mayor vida media y permiten administrarse una vez a la semana.

Los análogos de acción prolongada son sujetos a taquifilaxia (tolerancia) a su efecto inicial sobre el enlentecimiento del vaciamiento gástrico debido a la acción sostenida de la activación del receptor, motivo por el cual el efecto sobre el retraso del vaciamiento gástrico es menor que en los GLP-RA de acción corta ${ }^{38}$.

En los estudios con exenatide Lar denominados DURATION una dosis semanal de exenatide Lar fue comparada para determinar eficacia y seguridad con exenatide dos dosis diarias (DURATION 1), sitagliptina o pioglitazona (DURATION 2), insulina glargina (DURATION 3), metformina, pioglitazona y sitagliptina (DURATION 4), con liraglutide (DURATION 6). Si bien los resultados fueron beneficiosos con respecto a la reducción de $\mathrm{HbA} 1 \mathrm{c}$, ausencia de hipoglucemias y descenso de peso, fueron reportados episodios de molestias gastrointestinales $y$ reacciones locales en el sitio de inyección ${ }^{39-44}$.

Liraglutide tiene más efecto sobre la GA, efecto mediado por su estímulo sobre la célula beta. Se observó que tiene un efecto limitado a una hora en el retraso de vaciamiento gástrico luego de tres semanas de tratamiento. La acción de exenatide Lar es similar, a diferencia de exenatide que al ser administrado dos veces al día, no desensibilizaría al receptor ${ }^{35}$.

Liraglutide: los estudios LEAD fueron seis trabajos randomizados, cuya duración fue de entre 
26 y 52 semanas para evaluar la eficacia y seguridad con dosis de entre 0,6 y 1,8 mg, una vez al día solas o en combinación con ADOs en personas con diabetes tipo 2. También los resultados fueron alentadores en cuanto al descenso de HbA1c, peso corporal y tasa de hipoglucemias con respecto a sus comparadores ${ }^{45}$.

- Asociación de GLP-1 con insulina basal. Un tratamiento innovador ha sido la asociación de GLP-1 RA con insulina basal, que tiene como ventaja un mejor control de la GA y la GPP, con menor ganancia de peso y riesgo de hipoglucemia que con el régimen basal bolo. Otros argumentos a favor del uso de esta combinación es la disminución de la frecuencia de las inyecciones y que la dosis sea independiente del tipo de ingesta.

La asociación de esta terapia tiene efectos complementarios: el control de la hiperglucemia en ayuno por la insulina basal y el descenso de la glucemia postprandial por efecto del GLP-1 de acción corta.

Los GLP-1 RAs de acción prolongada actúan predominantemente sobre la GA y los niveles de glucagón, por lo cual los efectos de la terapia de combinación con estos fármacos y la insulina parecen ser mediados a través de acciones en puntos similares en el perfil diario de fluctuación del nivel de glucosa en el caso en ambos fármacos.

Un trabajo donde se evidencia el efecto complementario en la asociación entre un GLP-1 prandial como el lixisenatide e insulina, es el Get-Goal L, un estudio diseñado para evaluar eficacia y seguridad de lixisenatide vs. placebo asociado a insulina basal \pm metformina. Se incluyeron 495 pacientes DM2 con $\mathrm{HbA} 1 \mathrm{c}$ entre 7 y $10 \%$, en tratamiento con insulina $\geq 30$ unidades (glargina, detemir, NPH) \pm metformina. El seguimiento fue de 52 semanas. Con respecto al punto primario, en el grupo lixisenatide se observó un descenso de $\mathrm{HbA} 1 \mathrm{c}$ de $0,74 \%$ vs. $0,34 \%$ en el grupo placebo. Con un fuerte efecto en la glucemia postprandial a las 24 semanas (diferencia de medias [95\% Cl] vs. placebo: $-3,81$ $\mathrm{mmol} / \mathrm{L}$ (68,4 mg/dl) [-4.699, -2.925]; $p<0,0001)$. Lixisenatide redujo significativamente la dosis de insulina a las 24 semanas. Fue bien tolerado el lixisenatide asociado a insulina basal + metformina. Lixisenatide no incrementó significativamente la tasa de hipoglucemias vs. placebo ${ }^{46}$.

En la Tabla 5 se visualiza de forma rápida las características de los GLP-1 actualmente disponibles, y el modo de evaluar las ventajas y desven- tajas de cada uno a fin de elegir aquel que tenga las características apropiadas para cada paciente con $\mathrm{DM} 2^{47,48}$.

\begin{tabular}{|c|c|c|c|c|c|c|}
\hline GLP-1 & $\begin{array}{l}\text { Dura- } \\
\text { ción de } \\
\text { acción }\end{array}$ & $\begin{array}{l}\text { Mecanismo } \\
\text { de acción }\end{array}$ & Dosis & $\begin{array}{c}\text { Efecto } \\
\text { en PPG }\end{array}$ & $\begin{array}{l}\text { Efecto en } \\
\text { GPA }\end{array}$ & $\begin{array}{c}\text { Asociado } \\
\text { a otras } \\
\text { terapias }\end{array}$ \\
\hline $\begin{array}{l}\text { Exenatide } \\
\text { Byetta, } \\
\text { Amalyn } \\
\text { Pharma- } \\
\text { ceuticals } \\
(2006)\end{array}$ & $\begin{array}{l}\text { Corta } \\
\text { acción }\end{array}$ & $\begin{array}{l}\text { Prandial, } \\
\text { enlentence } \\
\text { el vacia- } \\
\text { miento } \\
\text { gástrico }\end{array}$ & $\begin{array}{l}\text { Dos } \\
\text { veces } \\
\text { por día } \\
\text { antes, } \\
\text { de la } \\
\text { comidas }\end{array}$ & $\begin{array}{l}\text { Fuerte } \\
\text { reduc- } \\
\text { ción }\end{array}$ & $\begin{array}{l}\text { Modesta } \\
\text { reducción }\end{array}$ & ADOS \\
\hline $\begin{array}{l}\text { Lira- } \\
\text { glutide, } \\
\text { Vyctoza, } \\
\text { Novo } \\
\text { Nordisk } \\
(2009)\end{array}$ & $\begin{array}{l}\text { Larga } \\
\text { acción }\end{array}$ & $\begin{array}{l}\text { No pran- } \\
\text { dial, me- } \\
\text { nor efecto } \\
\text { sobre el } \\
\text { vaciamien- } \\
\text { to gástrico }\end{array}$ & $\begin{array}{l}\text { Una vez } \\
\text { por día } \\
\text { indepen- } \\
\text { diente- } \\
\text { mente } \\
\text { de la } \\
\text { comida }\end{array}$ & $\begin{array}{l}\text { Mo- } \\
\text { desta } \\
\text { reduc- } \\
\text { ción }\end{array}$ & $\begin{array}{l}\text { Fuerte } \\
\text { reducción }\end{array}$ & $\begin{array}{l}\text { ADOs } \\
\text { y/o } \\
\text { análogos } \\
\text { lento } \\
\text { detemir }\end{array}$ \\
\hline $\begin{array}{l}\text { Lixisena- } \\
\text { tide } \\
\text { Lyxumia, } \\
\text { Sanofi } \\
(2013)\end{array}$ & $\begin{array}{l}\text { Corta } \\
\text { acción }\end{array}$ & $\begin{array}{l}\text { Prandial, } \\
\text { enlentece } \\
\text { el vacia- } \\
\text { miento } \\
\text { gástrico }\end{array}$ & $\begin{array}{l}\text { Una vez } \\
\text { por día } \\
\text { antes } \\
\text { de la } \\
\text { comida }\end{array}$ & $\begin{array}{l}\text { Fuerte } \\
\text { reduc- } \\
\text { ción }\end{array}$ & $\begin{array}{l}\text { Modesta } \\
\text { reducción }\end{array}$ & $\begin{array}{l}\text { ADOs } \\
\text { y/o } \\
\text { insulina }\end{array}$ \\
\hline
\end{tabular}

Tabla 5: Características diferenciales entre los análogos/ símiles de GLP-1. Adaptado de J. Vidal. European Endocrinology, 2013; 9(2):76-8147.

\section{CONCLUSIONES}

En el mundo viven aproximadamente $380 \mathrm{mi}-$ llones de personas con diabetes, lo cual convierte a esta enfermedad en una de las principales causas de muerte en la mayoría de los países desarrollados. Es sabido que la diabetes mal controlada se asocia al desarrollo de nefropatía, neuropatía, retinopatía, amputaciones y complicaciones macrovasculares. Aunque el control de la hiperglucemia en ayunas es necesario, suele ser insuficiente para conseguir un óptimo control glucémico.

Existe una fuerte asociación entre la glucemia postprandial y el aumento del riesgo cardiovascular, como así una asociación entre GPP y estrés oxidativo, inflamación, aumento del espesor miointimal carotídeo y disfunción endotelial, todos ellos marcadores de enfermedad cardiovascular. También hay datos que demuestran que la GPP se vincula a retinopatía, disfunción cognitiva en personas mayores con DM2 y a ciertos tipos de cáncer.

En la actualidad contamos con nuevas herramientas para lograr los objetivos glucémicos en las personas con diabetes, los análogos y símiles de GLP-1 de acción corta que tienen ventajas con respecto a los antidiabéticos orales. Los GLP-1 de 
acción corta, además de tener un impacto más preciso en la GPP sin provocar hipoglucemias, logran el descenso de peso, objetivo siempre buscado en pacientes diabéticos tipo 2 .

Cuando nos referimos al tratamiento de la DM2 es necesario evaluar cada paciente para elegir la terapéutica apropiada de acuerdo al comportamiento de la glucemia.

\section{REFERENCIAS}

1. Delahanty L, Simkins SW, Camelon K. Expanded role of the dietitian in the diabetes control and complications trial: implications for clinical practice. The DCCT Research Group. J. Am. Diet. Assoc. 1993 Jul; 93(7):758-64, 767.

2. Tight blood pressure control and risk of macrovascular and microvascular complications in type 2 diabetes: UKPDS 38. UK Prospective Diabetes Study Group. BMJ. 1998 Sep. 12; 317(7160):703-13.

3. Colclough H, Percy J, Benford M. Levels of FPG and HbA1c control and the relationship to BMI in T2D patients treated with basal insulin and OAD therapy, presented at the 2012 72nd Scientific Session at the American Diabetes Association Meeting , 8-12 June, Philadelfia, USA. Abstrac 2416 -PO,01-D.

4. Monnier L, Collete C, Dunseath GJ, et al. The loss of postprandial glycemic control precedes stepwise deterioration of fasting with worsening diabetes. Diabetes Care 2007; 30:263-9.

5. Ceriello A, Colagiuri S. Guideline for management of postmeal glucose. http://www.idf.org/webdata/docs/Guideline_PMG_final.pdf, 2007 (último acceso 22 de diciembre 2013).

6. Schrot R. Targeting plasma glucose: preprandial versus postprandial. Clinical Diabetes, 2004:22:169-72.

7. Parkin C. Is postprandial glucose control important? Is it practical in primary care settings? Clinical Diabetes, 2002; 20:71-6.

8. Erlinger TP, Brancati FL. Postchallenge hyperglucemia in a national sample of U.S. adults with type 2 diabetes. Diabetes Care, 2001; 24:1734-8.

9. Cavalot F, Pagliarino A, Valle M. Postprandial blood glucose predicts cardiovascular events and all-cause mortality in type 2 diabetes in a 14-year follow up: lessons from the San Luigi Gonzaga diabetes study. Diabetes Care, 2011; 34:2237-43.

10. Ferrarini E. Physiology of glucose homeostasis and insulin therapy in type 1 and type 2 diabetes. Endocrinol. Metab. Clin. N. Am. 41 (2012) 25-39.

11. Monnier L, Mas E, Ginet C, Michel F, Villon L, Cristol JP et al. Activation of oxidative stress by acute glucose fluctuations compared with sustained chronic hyperglycemia in patients with type 2 diabetes. JAMA 2006; 295(14):1681-1687.

12. Hasegawa G, Yamamoto Y, Zhi JG, et al. Daily profile of plasma $\% \mathrm{CoO} 10$ level, a biomarker of oxidative stress, in patients with diabetes manifesting postprandial hyperglycaemia. Acta Diabetol. $2005 ; 42(4): 179-181$.

13. Ross R. The pathogenesis of atherosclerosis: a perspective for the 1990s. Nature 1993; 362(6423):801-809.

14. Ceriello A, Falleti E, Motz E, Taboga C, Tonutti L, Ezsol Z et al. Hyperglycemia-induced circulating ICAM-1 increase in diabetes mellitus: the possible role of oxidative stress. Horm. Metab. Res. 1998; 30(3):146-149.

15. Ceriello A, Quagliaro L, Piconi L, Assaloni R, Da RR, Maier A et al. Effect of postprandial hypertriglyceridemia and hyperglycemia on circulating adhesion molecules and oxidative stress generation and the possible role of simvastatin treatment. Diabetes $2004 ; 53(3): 701-710$.
16. Kawano H, Motoyama T, Hirashima O, Hirai N, Miyao Y, Sakamoto $T$ et al. Hyperglycemia rapidly suppresses flow-mediated endothelium-dependent vasodilation of brachial artery. J. Am. Coll. Cardiol. 1999; 34(1):146-154.

17. Williams SB, Goldfine AB, Timimi FK, Ting $H H$, Roddy MA, Simonson DC et al. Acute hyperglycemia attenuates endothelium-dependent vasodilation in humans in vivo. Circulation 1998; 97(17):1695-1701.

18. Marfella R, Quagliaro L, Nappo F, Ceriello A, Giugliano D. Acute hyperglycemia induces an oxidative stress in healthy subjects. J Clin. Invest. 2001; 108(4):635-636.

19. DECODE Study Group, The European Diabetes Epidemiology Group. Glucose tolerance and cardiovascular mortality: comparison of fasting and 2-hour diagnostic criteria. Arch. Intern. Med .2001; 161:397-405.

20. Levitan EB, Song Y, Ford ES, et al. Is nondiabetic hyperglycemia a risk factor for cardiovascular disease? A meta-analysis of prospective studies. Arch. Intern. Med. 2004; 164:2147-2155.

21. Baltimore Longitudinal Study of Aging.

22. Tominaga $M$, Eguchi $H$, Manaka $H$, et al. Impaired glucose tolerance is a risk factor for cardiovascular disease, but not impaired fasting glucose. Diabetes Care, 1999 22:920-924.

23. Meigs JB, Nathan DM, D' Agostino RB, et al. Framingham Offspring Study. Fasting and postchallenge glycemia and cardiovascular disease risk: the Framingham Offspring Study. Diabetes Care 2002; 25:1845-1850.

24. Guideline for Management of Post Meal Glucose in Diabetes, IDF 2011, available in www.idf.org (último acceso 3 enero 2014).

25. American Diabetes Association. Standards of medical care in diabetes 2011. Diabetes Care 2011; 34. (Suppl. 1):S11-S61.

26. Hanefeld M, Fischer S, Julius $U$, et al. Risk factors for myocardial infarction and death in newly detected NIDDM: the Diabetes Intervention Study, 11-year followup. Diabetologia 1996; 39:1577-1583.

27. Esposito K, Giugliano D, Nappo F, et al. Regression of carotid atherosclerosis by control of postprandial hyperglycemia in type 2 diabetes mellitus. Circulation 2004; 110:214-21.

28. Chiasson JL, Josse RG, Gomis R, et al. STOP-NIDDM Trial Research Group JAMA. Acarbose treatment and the risk of cardiovascular disease and hypertension in patients with impaired glucose tolerance: the STOP-NIDDM trial. JAMA 2003, Jul 23; 290(4):486-94.

29. Patel YR, Kirkman MS, Considine RV, et al. Effect of acarbose to delay progression of carotid intima-media thickness in early diabetes. Diabetes Metab. Res. Rev. 2013 Oct; 29(7):582-91.

30. Stein SA, Lamos EM, Davis SN. A review of the efficacy and safety of oral antidiabetic drugs. Expert Opin 2013; 12(2):153-75.

31. Ceriello A. Point: postprandial glucose levels are a clinically important treatment target. Diabetes Care, 2010; 33 (8) 1905-1907.

32. Owens DR, Monnier L, Bolli GB. Differential effects of GLP-1 receptor agonists on components of dysglycaemia in individuals with type 2 diabetes mellitus. Diabetes Metabolism, 2013 Dec; 39(6):485-96.

33. Kendall DM, Riddle MC, Rosenstock J, et al. Effects of exenatide (exendin-4) on glycemic control over 30 weeks in patients with type 2 diabetes treated with metformin and a sulfonylurea. Diabetes Care, 28(5) 1083-1091.

34. De Fronzo RA, Okerson T, Viswanathan $P$, et al. Effects of exenatide versus sitagliptin on postprandial glucose, insulin and glucagon secretion, gastric emptying, and caloric intake: a randomized, cross-over study. Curr. Med. Res. Opin. 2008 Oct; 24(10):2943-52.

35. Lorenz M, Pfeiffer C, Steinsträsser A, Becker RH, Rütten H, Ruus $P$, Horowitz $M$, et al. Effects of lixisenatide once daily on gastric emptying in type 2 diabetes-relationship to postprandial glycemia. Regul. Pept. 2013;10;185:1-8. 
36. Bollim GB, Owens DR. Lixisenatide, a novel GLP-1 receptor agonist: efficacy, safety and clinical implications for type 2 diabetes mellitus. Diabetes Obes. Metab. 2013.

37. Rosenstock J, Raccah D, Koranyi L, et al. Efficacy and safety of lixisenatide once daily versus exenatide twice daily in type 2 diabetes inadequately controlled on metformin (Get-Goal-X), Diabetes Care 2013: 36; 2945-51.

38. Nauck MA, Kemmeries G, Holst et al. Rapid tachyphylaxis of the glucagon-like peptide 1-induced deceleration of gastric emptying in humans. Diabetes; 60 (5): 1561-1565.

39. Buse JB, Drucker DJ, Taylor KL, et al. DURATION-1: exenatide once weekly produces sustained glycemic control and weight loss over 52 weeks. Diabetes Care. 2010; 33(6):1255-61.

40. Wysham C, Bergenstal R, Malloy J, et al. DURATION-2: efficacy and safety of switching from maximum daily sitagliptin or pioglitazone to once-weekly exenatide. Diabet. Med. 2011; 28(6):705-14.

41. Diamant M, Van Gaal L, Stranks S, et al. Once weekly exenatide compared with insulin glargine titrated to target in patients with type 2 diabetes (DURATION-3): an open-label randomised trial. Lancet, 2010; 26;375:2234-43.

42. Russell-Jones D, Cuddihy RM, Hanefeld M, et al. Efficacy and safety of exenatide once weekly versus metformin, pioglitazo- ne and sitagliptin used as monotherapy in drug-naive patients with type 2 diabetes (DURATION-4): a 26-week double-blind study. Diabetes Care. 2012; 35(2):252-8.

43. Blevins T, Pullman J, Malloy J, et al. DURATION-5: exenatide once weekly resulted in greater improvements in glycemic control compared with exenatide twice daily in patients with type 2 diabetes. J Clin. Endocrinol. Metab. 2011 May; 96(5):1301-10.

44. Buse JB, Nauck M, Forst T, et al. Exenatide once weekly versus liraglutide once daily in patients with type 2 diabetes (DURATION-6): a randomised, open-label study. Lancet. 2013;381(9861):117-24.

45. Barnett AH. The role of GLP-1 mimetics and basal insulin analogues in type 2 diabetes mellitus: guidance from studies of liraglutide. Diabetes Obes. Metab. 2012; 14(4):304-14.

46. Get-Goal L.

47. J.Vidal. Lixisenatide. A new glucagon-like peptide 1 receptor agonist in the treatment of type 2 diabetes. European Endocrinology, 2013; 9(2):76-81.

48. Kapitza1 C, Forst 2, Coester HV, et al. Pharmacodynamic characteristics of lixisenatide once daily versus liraglutide once daily in patients with type 2 diabetes insufficiently controlled on metformin. 